\title{
Adquisición de Tecnología: Un Modelo de Gestión
}

\author{
Rafael Rincón Bermúez \\ Universidad EAFIT \\ rdrinconb@gmail.com
}

\author{
Guadalupe Peláez Ramírez \\ Politécnico Colombiano Jaime Isaza Cadavid \\ guapera802@gmail.com
}

Resumen: En la actualidad, las empresas basan su gestión en la utilización de la información, por lo cual necesitan el apoyo de los recursos tecnológicos para facilitar esta labor, sin importar la razón social o el sector productivo al que pertenezcan. Es por ello, que las Tecnologías de la Información juegan un papel muy importante para la toma de decisiones, brindando en el menor tiempo posible información organizada. Sin embargo, en muchas ocasiones por responder a necesidades internas o externas de manera precipitada, se adquieren tecnologías de información sin un adecuado proceso de análisis organizativo y establecimiento de requerimientos, lo que implica en algunos casos una errada selección de los proveedores o una adquisición de tecnología equivocada. Este modelo se fundamenta en unos referentes normativos tanto internos como externos, así como en unos componentes teóricos, asociados a cada una de las fases que lleva éste y que servirán para la comprensión del mismo. Además, el Modelo de Gestión para la Adquisición de Tecnologías de Información, servirá como base para llevar a cabo de forma organizada el proceso a seguir en la contratación de un servicio o producto en materia de Tecnologías de la Información.

Palabras clave: Adquisición de tecnología, tercerización, modelo de gestión, calidad, gestión de proyectos. 


\title{
Technology Acquisition: A Management Model
}

\begin{abstract}
Currently, the companies base their management on the use of the information, so that's why they need the support of technological resources to facilitate this work, regardless of the company name or the productive sector from which they belong. Therefore, information technology plays an important role in decision-making, providing information organized in the shortest time as possible. However, on many occasions to respond to internal or external needs precipitously, information technologies are acquired without an adequate organizational process of analysis and establishment of requirements, which in some cases means a wrong selection of suppliers or acquisition of wrong technologies. This model is based on internal and external policies as well as theoretical components associated with each of its phases, which will serve as an understanding of the model itself.
\end{abstract}

Keywords: Acquisition of technologies, outsourcing, management model, quality, suppliers, project management.

\section{Introducción}

La adquisición de tecnología cada vez cobra más importancia para las empresas de cualquier sector productivo u objeto social, pues la variable tecnológica, se ha convertido en elemento estratégico para su desarrollo y soporte fundamental en el desarrollo de sus operaciones y globalización, al facilitar la interacción y comunicación de los mercados de todo el mundo.

Sin embargo, en muchas ocasiones, por responder a necesidades internas o externas de manera precipitada, se adquieren tecnologías de información sin un adecuado proceso de definición y análisis de requerimientos, lo que implica en algunos casos selección de proveedores y adquisición de tecnología que no está relacionado efectivamente con el desarrollo del negocio.

El Modelo de Gestión de Adquisición de Tecnologías de la Información (MOGATI), que se propone, plantea llevar a cabo de forma organizada el proceso a seguir desde la definición hasta la contratación de un servicio o 
producto de $\mathrm{TI}$, integra en un proceso claramente definido los aspectos legales, los procesos internos de la organización y la calidad, tal que integrados propongan actividades, roles y productos que aseguren una selección de tecnología acorde con los requerimientos.

El Modelo se validó en el Politécnico Colombiano Jaime Isaza Cadavid, Institución Universitaria de carácter público, igualmente, se sometió a juicios de expertos de entidades públicas y privadas con el fin de verificarlo y ajustarlo.

En la sección 2 se describen los antecedentes del modelo propuesto; en la sección 3 se presenta el marco de referencia del modelo; en la sección 4 se describe el modelo propuesto y en la sección 5 se muestran las conclusiones y trabajos futuros.

\section{Antecedentes}

Las organizaciones y empresas de consultoría tienen definidos procesos de adquisición de $\mathrm{TI}$, que en alguna medida no integran todos los elementos de un proceso de esta naturaleza. Para el presente trabajo, se analizaron los modelos de gestión descritos por Amador y Márquez (2009), el modelo de gestión de una entidad financiera local, el modelo de adquisición de TIC de "la Caixa”, etapas del modelo de gestión estratégica de tecnología de Pedroza (2001), el modelo de gestión del Plan Estratégico de Tecnologías de la Información de Azurian (2012); todos estos proponen una secuencia lógica para la adquisición de TI, pero cada uno ajusta ese proceso a la arquitectura empresarial que han definido y a sus flujos internos de trabajo, convirtiendo a cada modelo en único, dejando a la organización como responsable de la ejecución o no de algunas actividades.

También se determinó como factor clave de la adquisición de TI, el aumento a nivel mundial de las inversiones en tecnología tanto en países desarrollados 
como en desarrollo, teniendo en cuenta que estos últimos están mejorando su acceso a los diferentes recursos, como la movilidad, la seguridad, los servicios

digitales, el almacenamiento de información, entre otros. Cifras como las de la consultora IDC (International Data Corporation) en su informe Latin America Predictions del mes de febrero de 2013, prevé que Latinoamérica, será la región con el crecimiento más rápido de TI en el mundo, teniendo en cuenta que el hardware equivaldrá al $66 \%$ del crecimiento, el software el $13 \%$ y los servicios el $21 \%$ restante, y que México, Brasil y Colombia serán los mercados de mayor crecimiento, y por ende, los que más adquieran tecnologías de la información. Sin embargo, en la actualidad se presentan obstáculos que deben ser tenidos en cuenta a la hora de invertir y adquirir tecnología, como déficit en infraestructuras, inestabilidad de las reglas institucionales, derechos de propiedad mal definidos, innovación tecnológica, entre otros. Por tal motivo, el fortalecimiento de las capacidades para organizar y estandarizar los procesos de identificación de necesidades tecnológicas se hace fundamental para su adquisición.

\section{Marco de Referencia}

En este trabajo, la gestión de tecnología se considera integrada por dos componentes principales: La Gestión Estratégica de TI y la Gestión Operativa de TI.

Gestión Estratégica de TI: Los elementos estratégicos hacen referencia a las políticas y objetivos que direccionan a la institución y definen las estrategias a seguir para alcanzar los resultados esperados.

Las tecnologías de la información se han convertido en una parte dominante para la realización de los procesos del negocio o misionales, y las instituciones de educación superior no son ajenas a este fenómeno, ya que no sólo tienen 
un enfoque operacional sino también estratégico, trayendo nuevos retos para los líderes institucionales, afectando no sólo las áreas administrativas sino también las académicas. La negociación de tecnología y su adquisición, ocurre cada vez con más frecuencia, y se ha vuelto más compleja y más crítica para el éxito del desarrollo de la organización, involucrando mayor número de personal con habilidades necesarias para negociar de forma satisfactoria.

La tecnología debe implementarse ordenadamente siguiendo los parámetros creados por la planeación estratégica en la Institución, identificando los recursos tecnológicos más importantes que agregarán valor y mejorarán la prestación del servicio, haciendo énfasis en permitir la aprehensión de nuevos conocimientos en todos los niveles del Politécnico. Este componente debe iniciar desde la estrategia institucional, plasmada en planes y políticas, principalmente relacionadas con las tecnologías de la información, y las necesidades macro a nivel tecnológico deben ser identificadas desde la definición de las metas y objetivos institucionales, que permitan su adecuado desarrollo.

A nivel institucional, este componente debe tener en cuenta, en las políticas para la Gestión del Proceso de Tecnología de la Información, lo siguiente:

- Los objetivos institucionales deben estar definidos y acordados antes de iniciar la adquisición de cualquier solución de TI.

- La adquisición e implementación de tecnología en la Institución debe ser un proceso planeado que responda a las necesidades y objetivos generales y soporte los procesos clave identificados. De otra forma, los costos de introducción, implementación e interoperabilidad pueden crecer de manera insostenible, al tiempo que el valor agregado de su uso se reduce significativamente. La arquitectura empresarial y tecnológica es el primer paso para poner a la tecnología en el lugar que más aporte, brindando soluciones a las unidades de gestión y procesos misionales.

- Construir estratégicamente la gestión tecnológica a partir de directrices institucionales. 
- Definir intenciones estratégicas en tecnología, partiendo de la arquitectura institucional.

- Identificar habilidades tecnológicas en todo el personal (actuales y necesarias).

- Determinar actores tecnológicos que puedan ser partícipes constantes en procesos de adquisición de tecnología, y que cuenten con las habilidades para ello.

- Explorar ambientes tecnológicos que puedan aportar valor a la Institución, tanto administrativa como académicamente.

- Pronosticar, analizar y auditar la tecnología actual como punto de partida para las mejoras futuras.

- Construir mapas tecnológicos para la planeación de nuevas adquisiciones y para la implementación de nuevas tecnologías, soportados en la arquitectura tecnológica existente.

Gestión Operativa de Adquisición de TI: Este componente propone un proceso para la adecuada adquisición de tecnología, que inicia con la identificación de necesidades desde cada unidad de gestión institucional, pasando por el reconocimiento de tecnologías que aporten soluciones a las necesidades identificadas, luego seleccionándolas, negociándolas, implementándolas, adaptándolas, utilizándolas y asimilándolas y, terminando en la transferencia de conocimiento y lecciones aprendidas, con el fin de mejorar los procesos definidos. Todo lo anterior, descrito en fases que contienen actividades, responsables y entregables que deberán estar alineados con la legislación externa, y normatividad interna que gobierna al Politécnico Colombiano Jaime Isaza Cadavid, por su naturaleza de Institución Universitaria de carácter público.

El modelo propuesto integra componentes teóricos como base para su fundamentación y como punto de partida para tener que integrar todos los elementos conceptuales en un esquema organizado, como lo muestra la Figura 1. 


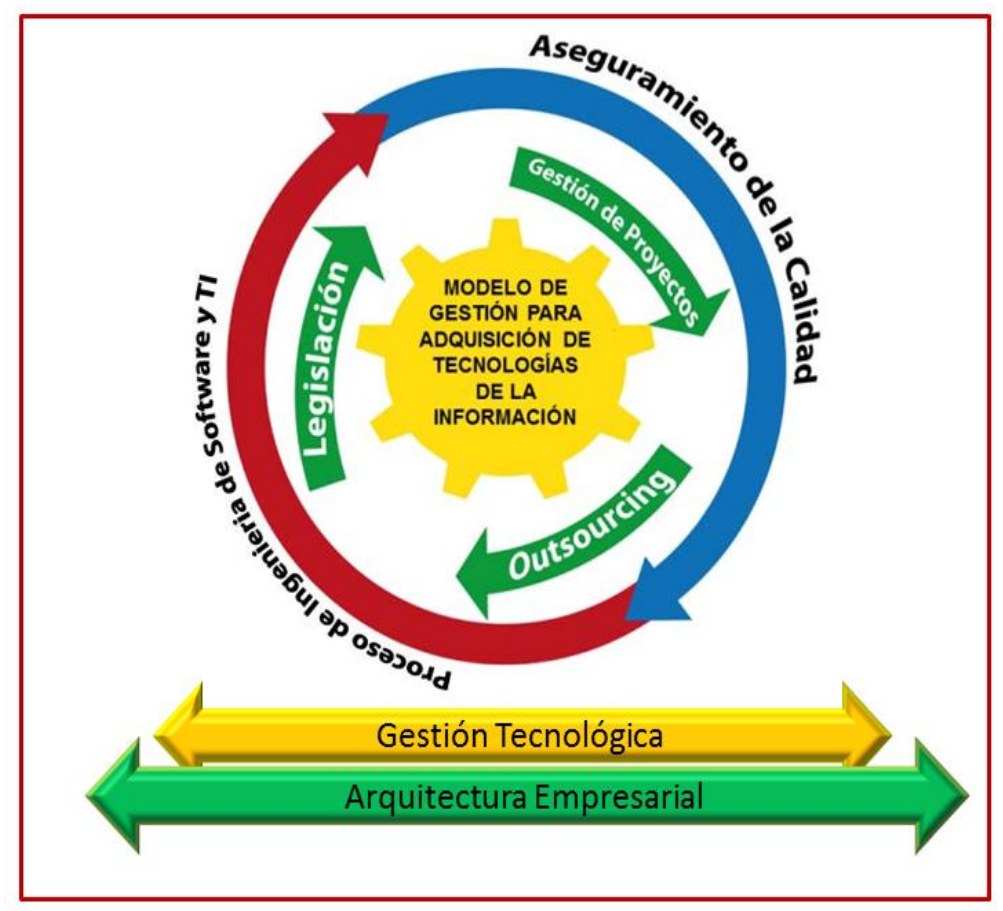

Figura 1. Componentes teóricos Modelo de Gestión para la Adquisición de Tecnologías de la Información.

En la figura anterior, la arquitectura empresarial relaciona el objeto institucional con las tecnologías de la información; la gestión de proyectos hace referencia a todas las actividades que se realizan para cumplir con un fin principal definido, en un tiempo establecido utilizando recursos tanto humanos como materiales y para el cual se deben tener presupuestados los costos en que se incurrirán; la gestión tecnológica es una práctica soportada en un conocimiento derivado del análisis y la interpretación de las observaciones del comportamiento del desarrollo tecnológico; el outsourcing es la transferencia de un proceso o una fase del negocio a un tercero especializado con el objetivo de agilizar y mejorar los procesos, tiene relación con este modelo dado que es frecuente que la variable TI sea tercerizada por las empresas en la actualidad. El Modelo debe tener como componente transversal el concepto de calidad, entendido como el nivel de cumplimiento de necesidades o expectativas establecidas por los clientes, a partir de unos rasgos o elementos diferenciadores de la organización. 


\section{Modelo de Gestión para la Adquisición de Tecnologías de la Información (MOGATI)}

Las fases propuestas por el modelo mostrado en la Figura2 son cuatro, y tienen un orden de ejecución, desde la primera y siguiendo continuamente hasta la cuarta, donde serán recogidas las lecciones aprendidas que retroalimentarán la Gestión Estratégica de TI, a partir de mejoras a tener en cuenta para futuras adquisiciones de tecnología.

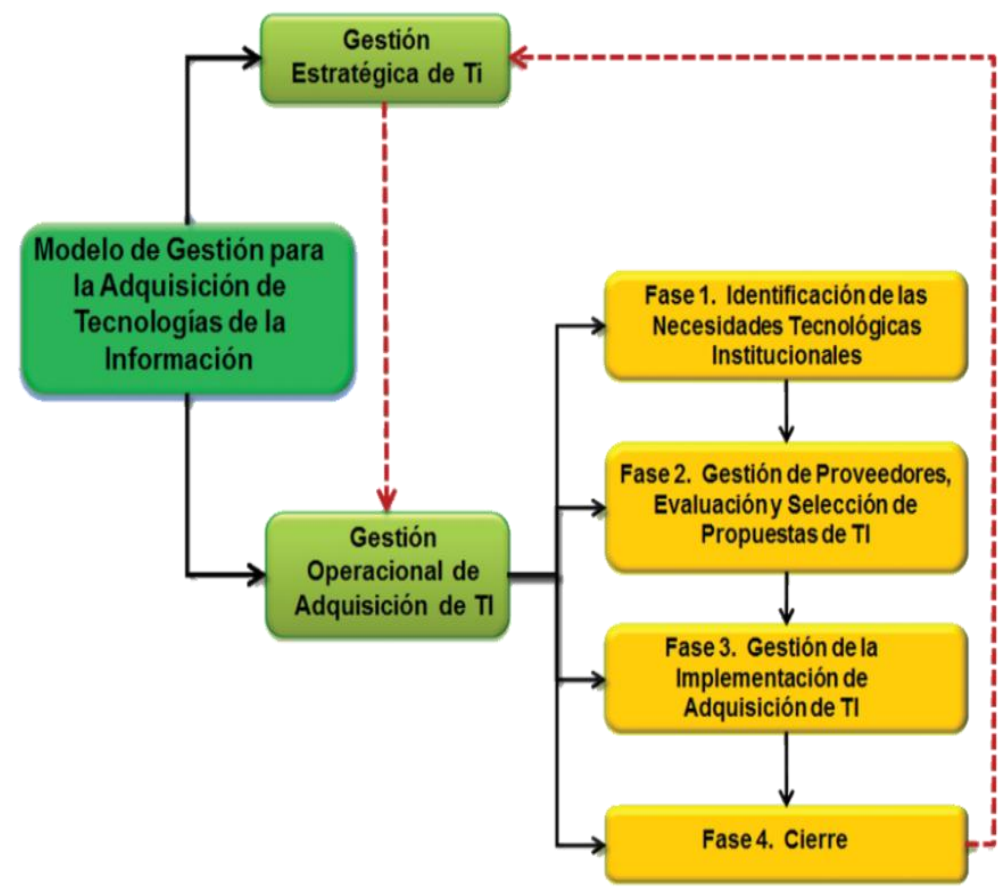

Figura 2. Modelo de Gestión para la Adquisición de Tecnologías de la Información para el Politécnico Colombiano Jaime Isaza Cadavid. 


\section{Fase 1. Identificación de las necesidades tecnológicas institucionales.}

Esta es la primera fase del Modelo de Gestión para la Adquisición de Tecnologías de la Información, y en ella se propone que cada unidad de gestión al identificar una necesidad de mejora, analice si ésta se puede solucionar a partir de la adquisición de tecnología o mejorando la existente, realizando un análisis que contenga los siguientes aspectos: Qué, por qué, para qué, cuándo, dónde y cómo, reflejados en la definición de requerimientos. Con la definición de requerimientos se expresa la necesidad que tiene la unidad de gestión o la Institución para adquirir una tecnología, donde se especifique de forma clara y precisa los aspectos más relevantes para la adquisición. Para realizar tal definición es necesario tener muy claras las necesidades que originan el requerimiento a nivel Institucional.

Es importante tener conocimiento de las empresas que proveen el producto $u$ ofrecen los servicios que se necesitan, con el fin de no sesgar el estudio por no contar con suficiente información de peso para establecer el presupuesto.

Esta fase aplica desde la definición de las necesidades tecnológicas hasta la identificación de las tecnologías de la información potenciales y que ofrecerán soluciones a estas necesidades.

\section{Fase 2. Gestión de Proveedores y Evaluación de Propuestas de TI.}

La segunda fase del Modelo de Gestión para la Adquisición de Tecnologías de la Información propone que se realice un proceso claro de evaluación de propuestas y selección de proveedores, teniendo como punto de partida la descripción detallada de los requerimientos especificados en la Fase 1, y elaborando a partir de ellos, una solicitud de propuestas o RFP (en inglés: Request For Proposal) que contendrá las bases para que los oferentes o 
proveedores potenciales realicen sus propuestas técnicas, funcionales, de proyecto y económicas, de la tecnología que se requiere adquirir, todo enmarcado bajo la naturaleza jurídica de la organización, y obedeciendo a las directrices definidas en los manuales internos de la compañía si los hay.

La selección del proveedor de la solución de TI está compuesta por el análisis formal de las propuestas de los proveedores y el análisis de las diferentes modalidades de adquisición. Esta selección debe responder a los planes, proyectos y programas que desarrollan las diferentes áreas de la Institución, en relación con la misión y la visión, ajustados a los Planes de Desarrollo, la programación presupuestal, así como al efectivo y permanente mejoramiento de la calidad de los procesos.

Esta fase aplica desde el refinamiento de los requerimientos definidos en la fase 1, hasta la selección del proveedor o proveedores de la tecnología a adquirir que dará solución a la necesidad planteada por la Unidad de Gestión involucrada.

\section{Fase 3. Gestión de la Implementación de Adquisición de TI.}

Para la tercera fase del Modelo propuesto, se describen unas actividades de manera general, sin entrar en detalles, ya que la fase de implementación variará, de acuerdo con la tecnología adquirida.

Esta fase inicia con la planificación de la implementación de la tecnología que se adquirió, y para esto debe existir una comunicación constante y clara entre el supervisor asignado al contrato y el proveedor seleccionado, y culmina con la implementación de la tecnología.

Para el buen funcionamiento del proyecto en esta fase, es necesario mantener un registro de la información comunicada y las decisiones tomadas. Asimismo, 
todos los documentos relativos al proyecto y sus versiones, contratos, todos estos documentos almacenados y publicados, de manera que pueden ser consultados por cualquier miembro del equipo.

Hay que tener en cuenta que durante el ciclo de vida del contrato pueden haber cambios y reclamaciones, así como nuevas oportunidades de desarrollo. El principal objetivo tras la concesión de un contrato es el control del riesgo. Se debe mantener en todo momento el control sobre el proyecto y asegurarse que se cumplen los requisitos de tiempo y costos.

Como resultado de esta fase se elabora un documento detallado de la arquitectura e instalación de la solución, componentes, soluciones a problemas que se puedan presentar, futuras actualizaciones, información de contactos para mantenimiento y mejoras, entre otros.

Esta fase aplica desde el inicio de toda la gestión para la implementación de la tecnología adquirida hasta su instalación y puesta a punto.

\section{Fase 4. Cierre.}

Esta es la última fase del Modelo y en ella es necesario definir las actividades relacionadas con la finalización del contrato e interacciones requeridas para establecer y cerrar todo el acuerdo contractual establecido para el proyecto, así como aquellas que respaldan el cierre administrativo. Esta fase implica tanto la verificación final de la tecnología implementada (todo el trabajo completado de forma correcta y satisfactoria) y la actualización de todos los registros del contrato para reflejar los resultados finales para su uso futuro, además, el análisis del proceso para gestión del conocimiento e identificación de acciones de mejora. Algunos aspectos que deben ser tenidos en cuenta en esta fase, son:

- Verificar el cumplimiento del contrato (desde su alcance). 
- Registrar adecuadamente la obtención de informes, autorizaciones y demás documentos derivados.

- Resolución de diferencias.

- Aseguramiento del cumplimiento de las obligaciones posteriores.

- Registro de toda la documentación, así como lecciones aprendidas.

Antes de la finalización del contrato, se debe revisar el mismo para encontrar aquellos aspectos en los cuales alguna de las partes debe mantener unas responsabilidades posteriores. Algunas de ellas pueden referirse a:

- Protección de la propiedad intelectual.

- Licencias.

- Servicios de mantenimiento, soporte y garantía.

- Actualizaciones de producto, nuevas versiones.

- Conservación de la información y los registros.

En esta fase de cierre, es fundamental que se realice el despliegue formal de la tecnología adquirida, mediante una reunión de inicio en producción de la solución comprada e implementada, con todos los involucrados en la misma. Esta fase aplica desde la planeación de la capacitación y entrenamiento del personal hasta la recolección y transferencia de lecciones aprendidas, que servirán como un componente de mejoramiento para la Gestión Estratégica de TI.

\section{Caso Politécnico Colombiano Jaime Isaza Cadavid}

El Politécnico Colombiano Jaime Isaza Cadavid como Institución Universitaria de carácter público, tiene las particularidades definidas en la Tabla 1. 


\section{LEGAL}

Ley 80 de 1993. Por la cual se expide el Estatuto General de Contratación de la Administración Pública.
Ley 1150 de 2007. Por medio de la cual se introducen medidas para la eficiencia y la transparencia en la ley 80 de 1993 y se dictan otras disposiciones generales sobre la contratación de recursos públicos.

\section{NORMATIVIDAD INSTITUCIONAL Y OTRAS}

Políticas de Informática Corporativa. Acuerdo 09 de septiembre de 2010

Manual de Contratación Politécnico Colombiano Jaime Isaza Cadavid - MLH01.

Plan de Desarrollo Institucional 2011-2016. "Una nueva visión para una nueva época"

\section{ASEGURAMIENTO DE LA CALIDAD (SISTEMA INTEGRADO DE GESTIÓN)}

Norma Técnica de Calidad en la Gestión Pública. NTCGP 1000:2009

Proceso de Tecnología de la Información

- Caracterización del proceso

- PTH02 - Procedimiento para proveer y administrar sistemas de información. V4.

- PTH07 - Procedimiento para proveer software específico. V1.

- PTP01 - Procedimiento para el análisis, desarrollo y mantenimiento de sistemas de información. V3

\section{GESTIÓN DE PROYECTOS}

- Metodología del Marco Lógico. Departamento Nacional de Planeación.

\section{Proceso de Logística}

- Caracterización del proceso

- PLH01 - Procedimiento para la Adquisición de Bienes y Servicios. V14

- PLH21 - Procedimiento para la contratación directa. V4

- $\quad$ LH01 - Ingreso y actualización de proveedores. V5

\section{ESTÁNDARES DE CALIDAD RELACIONADOS CON TI}

CMMI para Adquisición (CMMI ACQ). V. 1.3

- Guía de los Fundamentos para la Dirección de Proyectos. Guía PMBOK. Cuarta Edición.

Tabla 1. Marco de Referencia del Modelo de Gestión para Adquisición de TI, PCJIC. 
En ese contexto, el modelo propuesto permitió gestionar un proceso desde la fase de identificación de necesidades tecnológicas hasta la gestión de proveedores y evaluación de propuestas de TI, evidenciando con ello que el modelo se ajusta a los requerimientos del Politécnico.

\section{Conclusiones y Trabajos Futuros}

Se diseñó y se puso en ejecución un modelo de gestión, que tuviera en cuenta las buenas prácticas en la gestión de proyectos utilizadas por el sector privado, y las condiciones legales, normativas y de calidad del Politécnico Colombiano Jaime Isaza Cadavid.

La experiencia en las etapas iniciales del proceso de implantación del modelo desarrollado permite inferir que la gestión de procesos, esté fuertemente ligada con la cultura de las entidades y con la gestión del cambio, que en cada una de ellas se coordine. Por lo tanto, no depende única ni exclusivamente de la racionalidad del modelo.

El modelo implementado ha permitido incorporar mejoras para optimizar los procesos, pero si las unidades de gestión no son flexibles a los cambios que presenta el entorno, no podrán mejorar continuamente, lo que dificultaría el avance general de la Institución en materia tecnológica.

En cada una de las fases propuestas en el modelo de adquisición de TI, la Institución tuvo en cuenta la importancia de contar con expertos, tanto internos como externos, que sirvieran de apoyo a un proceso efectivo de selección de la tecnología a adquirir. 


\section{Referencias}

Agudelo M., Elena, Niebles A., Lina María y Gallón L., Luciano. (2005). La Gestión Tecnológica como Herramienta de Planeación Estratégica y Operativa para las Unidades de Información. Revista Interamericana de Bibliotecología, 89 - 114.

Amador, Belkys y Márquez, Alexandra. (2009). Un Modelo Conceptual para Gestionar la Tecnología en la Organización. Revista Espacios, 7 - 21

Benavides Velásquez, Carlos y Quintana García, Cristina. (2007). Un Modelo para la Gestión Estratégica de los Recursos Tecnológicos. Revista Economía Industrial, $195-206$.

Builes Vélez, Carlos Alberto. (2008). Notas acerca de la Ingeniería de Software. Medellín: Editorial Artes y Letras.

Carroz, Darnila. (2005). Modelo de Gestión Estratégica para el Desarrollo de Capacidades Tecnológicas. Revista Compendium, 5 -19.

Castillo Giraldo, Edwin Marino. (2006). Aseguramiento de la Calidad en el Proceso Delegado de Desarrollo de Software. Tesis de Ingeniería de Sistemas, Universidad EAFIT, Medellín.

COTEC, Fundación Española para la Ciencia y Tecnología. (2010). Análisis de Patrones en el Proceso de Transferencia de Conocimiento y Tecnología. Modelo de Transferencia de Tecnología y Conocimiento.Madrid: Autor.

COTEC, Fundación Española para la Ciencia y Tecnología. (1999). Pautas Metodológicas en Gestión de la Tecnología y de la Innovación para Empresas. Madrid: Autor.

Díaz Spataro, Mariana. (2012). Outsourcing, guía práctica de técnicas y estrategias. Bogotá: Ediciones de la U.

Gay, Aquiles. (1994). La Tecnología, el Ingeniero y la Cultura. Capítulo 1: La Ciencia, la Técnica y la Tecnología. Ediciones Tec. Córdoba, Argentina.

Hernández, René. (2011). Competencias para la Gestión de la innovación en la Formación Gerencial. Revista Formación Gerencial, 217 - 234.

Hidalgo Nuchera, Antonio. (1999). La Gestión de la Tecnología como Factor Estratégico de la Competitividad Industrial. Revista Economía Industrial, 43 - 54. 
IDC, International Data Corporation. (2012). Top 10 Predictions. IDC Predictions 2013: Competing on the 3rd Platform. MA: Autor.

Miranda, Juan José. (2010). El Desafío de la Gerencia de Proyectos. Bogotá: MM editores.

Ochoa Ávila, Migdely, Valdés Soa, Mario y Quevedo Aballe, Yovanni. (2007). Innovación, Tecnología y Gestión Tecnológica. Revista Cubana de los Profesionales de la Información y de la Comunición en Salud, (s/p).

Pantaleo, Guillermo. (2011). Calidad en el Desarrollo de Software. Buenos Aires: Alfaomega.

Pedroza, Álvaro. (2001). Modelo para la Gestión Estratégica de Tecnología. Revista Universidad Eafit, 23 - 37.

Pereña Brand, Jaime. (1996). Dirección y Gestión de Proyectos, (2da.Edición). Madrid: Ediciones Díaz de Santos.

Ramírez González, Leonardo. (2011). Arquitectura Empresarial, mitos y realidades. Encuentro ACIS, XXXI Salón de Informática, Bogotá, 12 - 14 de septiembre, (paper).

Sistema Integrado de Gestión. (2011). CPL07 - Caracterización del Proceso Gestión Logística V.6, Politécnico Colombiano Jaime Isaza Cadavid, Medellín: Intranet Sinapsis.

Sistema Integrado de Gestión. (2011). CPT11 - Caracterización del Proceso Gestión Tecnología de la Información V.6, Politécnico Colombiano Jaime Isaza Cadavid, Medellín: Intranet Sinapsis.

Sistema Integrado de Gestión. (2009). MLHO1 - Manual de contratación V.2, Politécnico Colombiano Jaime Isaza Cadavid, Medellín: Intranet Sinapsis.

Sistema Integrado de Gestión. (2011). PLHO1 - Procedimiento para la Adquisición de Bienes y/o Servicios - Estudio previo V.14, Politécnico Colombiano Jaime Isaza Cadavid, Medellín: Intranet Sinapsis.

Sistema Integrado de Gestión. (2009). PTH02 - Procedimiento para Proveer y Administrar los Sistemas de Información V.04, Politécnico Colombiano Jaime Isaza Cadavid, Medellín: Intranet Sinapsis.

Sistema Integrado de Gestión. (2011). PTH07 - Procedimiento para Proveer Software Específico V.01, Politécnico Colombiano Jaime Isaza Cadavid, Medellín: Intranet Sinapsis. 
Software Engineering Institute. (2011). CMMI for Acquisition (CMMI-ACQ) V. 1.3, Carnegie Mellon University, Pittsburgh.

Vega González, Luis Roberto. (2006). Modelo del Ciclo de Vida de un Proyecto de Gestión Tecnológica y Vinculación en un Centro de I+D Universitario. I Congreso Iberoamericano de Ciencia, Tecnología, Sociedad e Innovación, Universidad Nacional Autónoma de México, Ciudad de México, 19 - 23 de julio, (paper). 


\section{Notas biográficas}

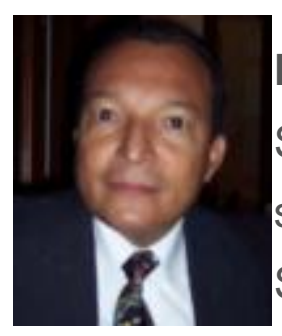

Rafael David Rincón Bermúez obtuvo el título de Maestría en Sistemas de Calidad del ITESM, Campus Monterrey de México, y se desempeña como profesor Titular del Departamento de Sistemas de la Universidad Eafit de Medellín, Colombia. Consultor en Mejora de Procesos de Negocio, Implementación de Sistemas, Modelos y Estándares de Calidad de Software, como CMMI.

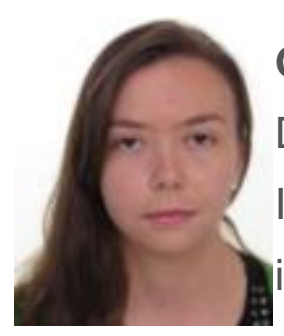

Guadalupe Peláez Ramírez es Tecnóloga en Sistematización de Datos y actualmente, estudiante de pregrado de Ingeniería Informática del Politécnico Colombiano Jaime Isaza Cadavid; igualmente se ha desempeñado como auxiliar de docencia en el área de Ingeniería de Software y docente de cátedra del proyecto de los Consultorios Tecnológicos en el Politécnico. En su trabajo como analista de datos en la Oficina Asesora de Planeación, su labor se fundamenta en la automatización de gestión de procesos y monitoreo de indicadores del Sistema de Calidad.

\section{(c) (i) (2)(2)}

Esta obra está bajo una licencia de Creative Commons Reconocimiento-NoComercial-Compartirlgual 2.5 México. 